See discussions, stats, and author profiles for this publication at: https://www.researchgate.net/publication/282711526

\title{
Multi-angle based lively sclera biometrics at a distance
}

Article · January 2015

DOI: 10.1109/CIBIM.2014.7015439

CITATIONS

4 authors, including:

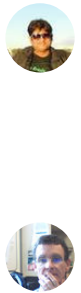

Abhijit Das

University of Southern California

42 PUBLICATIONS 300 CITATIONS

SEE PROFILE

Miguel A. Ferrer

Universidad de Las Palmas de Gran Canaria

281 PUBLICATIONS 2,970 CITATIONS

SEE PROFILE

Some of the authors of this publication are also working on these related projects:

Project $\quad$ COST Action CA15225 - Fractional-order systems - analysis, synthesis and their importance for future design View project

CNN on Degraded Images View project
READS

52

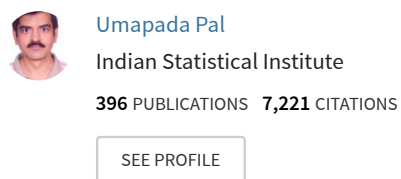




\title{
Multi-angle Based Lively Sclera Biometrics at a Distance
}

\author{
Abhijit Das ${ }^{\mathrm{a}}$ \\ ${ }^{a}$ Institute for Integrated and Intelligent Systems, Griffith University, Queensland, Australia \\ Email: abhijit.das@griffithuni.edu.au
}

\begin{abstract}
This piece of work proposes a liveliness sclera-based eye biometric validation and recognition technique at a distance. The images in this work are acquired by a digital camera in the visible spectrum at varying distance of about 1 meter from the individual. Each individual during registration as well as validation is asked to look straight and move their eye ball up, left and right keeping their face straight to incorporate liveliness of the data. At first the image is divided vertically into two halves and the eyes are detected in each half of the face image that is captured, by locating the eye ball by a Circular Hough Transform. Then the eye image is cropped out automatically using the radius of the iris. Next a C-means-based segmentation is used for sclera segmentation followed by vessel enhancement by the Haar filter. The feature extraction was performed by patch-based Dense-LDP (Linear Directive Pattern). Furthermore each training image is used to form a bag of features, which is used to produce the training model. Each of the images of the different poses is combined at the feature level and the image level. The fusion that produces the best result is considered. Support Vector Machines (SVMs) are used for classification. Here images from 82 individuals are used and an appreciable Equal Error Rate of $0.52 \%$ is achieved in this work.
\end{abstract}

Keywords-Biometric; Sclera Vessels Patterns; Liveliness, of data: Eye movement: D-LDP; SVM; Bag of features, Adaptive Histogram Equalization; Discrete Meyer Wavelet.

\section{Introduction}

Nowadays biometrics is believed to be the most reliable technique in regards to security for personal identification. Biometrics have enjoyed a large amount of research in the last few decades in every domain of research, but due to spoofing and other biometric attack it is becoming necessary to produce such system that can mitigate those attack. One of the techniques to minimize this treat is by proving the liveliness of the data.

Another interesting aspect of research in biometrics that is highly encouraged and interesting is biometrics at a distance, which is often required to identify an individual from a distance in a stipulated security environment. Eye biometrics like iris, sclera and retina are one of the most reliable biometric, as they are highly protected part of the eye, which is highly random for each individual, they do not change over time and they even varies for left and right eye of each individual [1], [2]. Among the ocular biometric iris is the most reliable one, this biometrics also poses few disadvantages. The capture of iris images requires high co-operation from the user since an off-axis iris image can deteriorate the system performance. This scenario becomes quite tough for people with squint eyes.

So, sclera biometric can be alternative in this regards which can be combining with iris biometric to increase its usefulness in non-ideal iris recognition scenario. Therefor multi-modal iris recognition using iris and sclera was proposed in literature, in these systems the images were captured in visible spectrum. Such systems can be spoofed easily by scanning a high resolution image or high definition video in front of the sensor. So it necessary to build liveliness based multi-modal eye biometric system.

In order to establish the concept of liveliness based multi-modal eye biometric system, it is first necessary to access that whether liveliness can be gained from a sclera biometric system. Even to date, sclera biometric is relatively less studied and little is known regarding its usefulness. To our knowledge, the first recognized work on sclera biometrics is recorded in [3]. Work on multi-angled sclera recognition [4-5]. A survey on sclera recognition is described in [9]. Few advance pieces of work can be found in $[6-8,13-18$, and 20]. To date, this biometric is relatively less studied and little is known regarding its usefulness

As mentioned above major problem in eye recognition is the spoofing with high resolution photographs. One of the most popular methods to overcome the threat of physical spoofing of biometric samples is the aliveness detection. The aliveness detection methods at the acquisition stage can be classified into the following three categories: 1) Intrinsic properties of a living body as physical (density, elasticity), electrical (capacitance, etc.), spectral (reflectance and absorbance), visual (colour, etc.) and analysis of body fluids (DNA, etc.), 2) Involuntary signals of living body as pulse, blood pressure, hippos, etc., and 3) 
Bodily response to external stimuli which require the user cooperation by instance smiling or blinking as it is done in face recognition which looks an easy and effective countermeasure for eye biometric spoofing

So, to mitigate the problem an initiative is taken in this piece of work for liveliness in eye biometrics for contactless sclera biometric: the user is required to look at different random angle each time approach to the biometric scheme. Two advantages are obtained: $1^{\text {st }}$. we require a body response for aliveness detection, and $2^{\text {nd }}$. Looking to left, right or up the portion of sclera shown is different. So, we can require a different sclera angle in case of not being sure of user identity.

This work proposes a whole biometric system for personal identification based on sclera vessels at distance with lively data. Here sclera segmentation was performed by Fuzzy C-means clustering. A new preprocessing approach for vein highlighting is proposed here by the Haar wavelet. Sclera feature extraction based on the Dense Local Directional Pattern (D-LDP) is also new in the literature. Support Vector Machines (SVMs) are used for classification.

The organization of the paper is as follows: - Section 2 explains the proposed Capturing technique, segmentation approach, preprocessing of the sclera images, followed by the sclera vessel enhancement process, feature extraction and classification. In Section 3, the experimental details are described and Section 4 draws the overall conclusions.

\section{Proposed Approach}

In this section, the proposed image capturing technique, sclera segmentation process, a sclera vein enhancement technique and feature extraction of sclera texture patterns are explained, and this is finally followed by the classification technique.

\subsection{Image capturing technique}

The images are acquired by digital camera in visible spectrum at a distance of about 1 meter from the individual as shown figure 1 (a). A framework of the image capturing technique is given below 1(b).

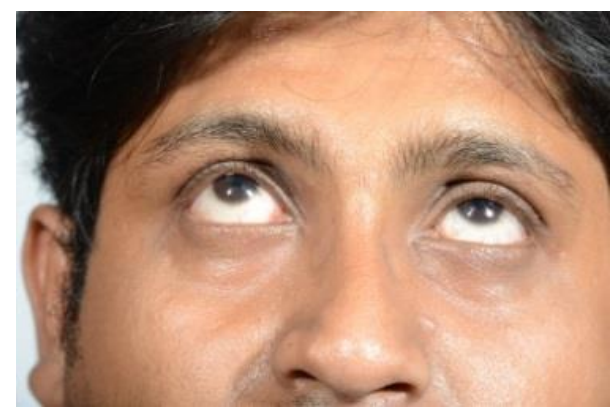

(a)

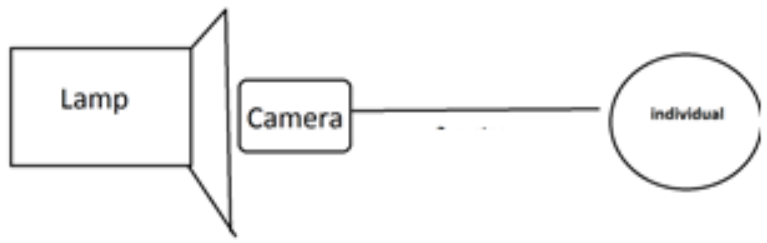

(b)

Figure 1: (a) Image capture at distance. (b). A framework of the image capturing technique

At first the image is divided vertically in to two half as shown in figure 2.

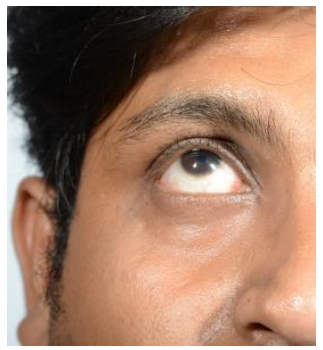

(a)

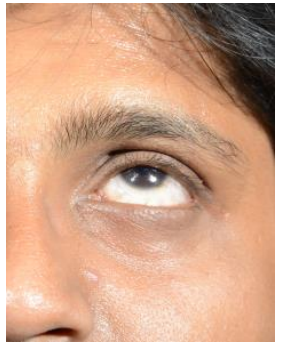

(b)
Figure 2: Two half of the face image.

The eyes are detected in each half of the face image that is captured, by locating the eye ball through the circular Hough transform as shown in Figure 3 below.

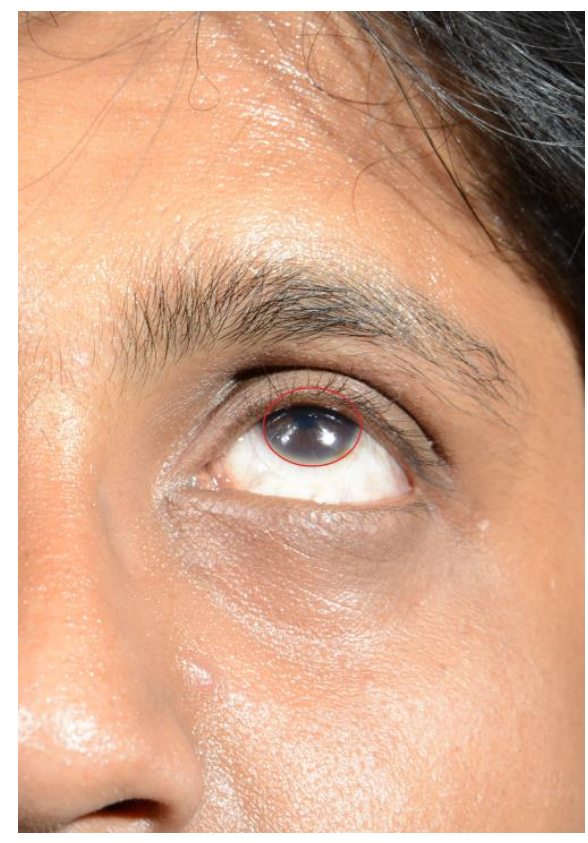

Figure 3: Hough Circle detected the eye ball 
The images are cropped automatically by calculating the radius of the eye ball as shown in figure below.

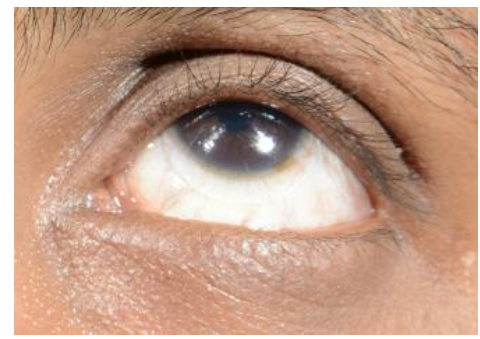

Figure 4: An automatically cropped image.

Each individual during registration as well as validation is asked to look straight and move their eye ball up, left and right keeping their face straight to incorporate liveliness of the data. The images of one individual are given in Figure 5 below.

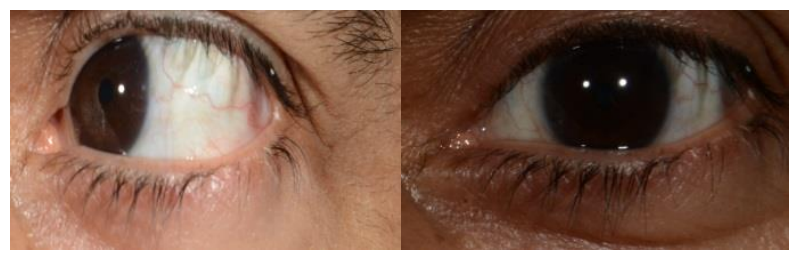

(a)

(b)

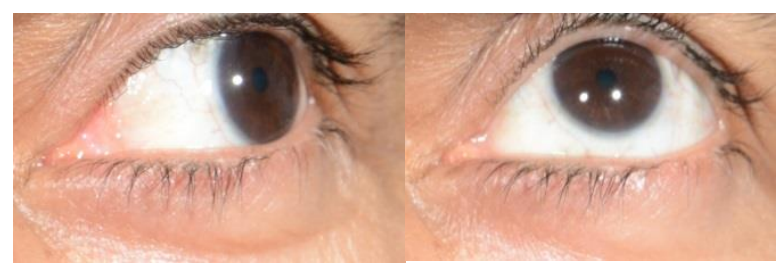

(c)

(d)

Figure 5: Images at different angle

\subsection{Sclera Segmentation}

. Segmentation is the first step for most biometric related research. Similarly, here accurate segmentation of the sclera region is very important, otherwise, an incorrect segmentation can reduce the pattern available, other way it can also introduce other patterns such as eyelashes and eyelids. Here sclera segmentation is performed by a Fuzzy $\mathrm{C}$-means clustering-based segmentation as proposed in [20]. The Segmented sclera images are shown in the below figure.

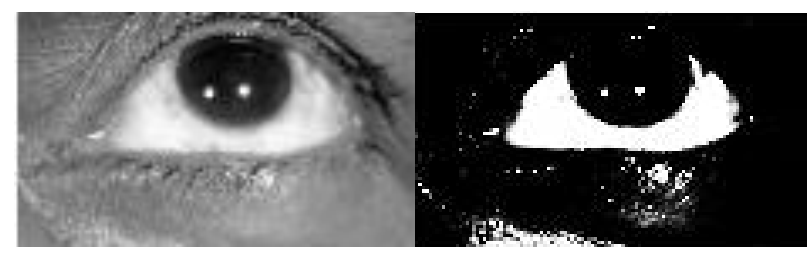

(a)

(b)

Figure 6: (a)The gray image of an eye image and (b) the mask created for it by c-means.

\subsection{Sclera vessel structure enhancement}

The vessels in the sclera are not prominent, so in order to make them clearly visible, image enhancement is required. Adaptive histogram equalization [21] was performed with a window size of $42 \times 42$ on the blue channel of the sclera image (as the sclera vessel patterns are most prominent in the blue channel as shown in figure 7(c)) to make the vessel structure more prominent as shown in Figure 8(a).

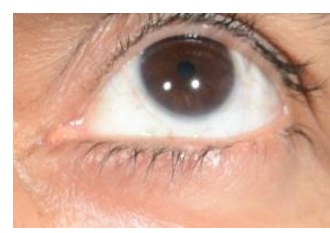

(a)

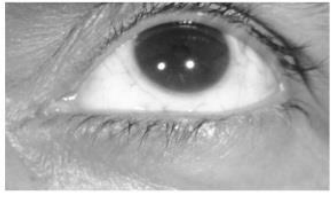

(c)

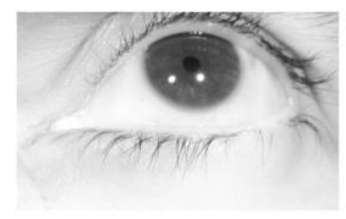

(b)

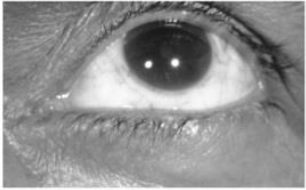

(d)
Figure 7 (a) The original RGB image, (b) The red channel component of (a), (c) The green channel component of (a), and (d) blue channel component of (a),

Farther Discrete Haar wavelet [22] was used to enhance the vessels pattern. Low pass reconstruction of the above mention filter was used to enhance the image. Figure 8(b) shows the vessel enhanced image after filter been applied.

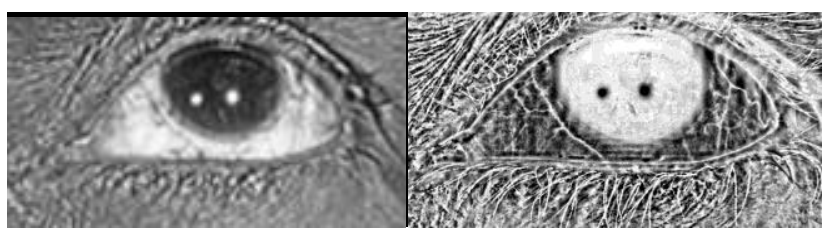

(a)

(b)

Figure 8: (a) Adaptive histogram equalization of sclera image. (b) The vessel enhanced image. 


\subsection{Feature Extraction Method}

A local descriptor method applied in this paper is the Local Directional Pattern (LDP), which computes the edge response values in different directions and uses these to encode the image texture [11].Considering the relative edge response values in different directions, the proposed LDP feature encodes the local neighborhood property of image pixels with a binary bit sequence. Sclera feature extraction based on Dense Local Directional Pattern (D-LDP) was performed here. D-LDP patch descriptors of each training image are used to form a bag of features, which was used to produce the training model. For extracting patch descriptors of each image, it is divided into four bins and eight orientations with $22 \times 22$ locations of $9 \times 9$ patch sizes. Here histogram of bin size 512, for of each of this patches are calculated. The entire eight orientations mask (as shown below ) of LDP is used here for calculating the LDP for each block in each scale.

$$
\begin{aligned}
& {\left[\begin{array}{ccc}
-3 & -3 & 5 \\
-3 & 0 & 5 \\
-3 & -3 & 5
\end{array}\right]\left[\begin{array}{ccc}
-3 & 5 & 5 \\
-3 & 0 & 5 \\
-3 & -3 & -3
\end{array}\right] \quad\left[\begin{array}{ccc}
5 & 5 & 5 \\
-3 & 0 & -3 \\
-3 & -3 & -3
\end{array}\right]\left[\begin{array}{ccc}
5 & 5 & -3 \\
5 & 0 & -3 \\
-3 & -3 & -3
\end{array}\right]} \\
& \text { Fast } M_{0} \quad \text { North Fast } M_{1} \text { North } M_{2} \text { North West } M \\
& {\left[\begin{array}{ccc}
5 & -3 & -3 \\
5 & 0 & -3 \\
5 & -3 & -3
\end{array}\right]\left[\begin{array}{ccc}
-3 & -3 & -3 \\
5 & 0 & -3 \\
5 & 5 & -3
\end{array}\right]\left[\begin{array}{ccc}
-3 & -3 & -3 \\
-3 & 0 & -3 \\
5 & 5 & 5
\end{array}\right]\left[\begin{array}{ccc}
-3 & -3 & -3 \\
-3 & 0 & 5 \\
-3 & 5 & 5
\end{array}\right]} \\
& \text { West } M_{4} \quad \text { South West } M_{4} \text { South } M_{6} \quad \text { South East } M \text {, }
\end{aligned}
$$

Figure 9: Eight Kirsch masks used to get the edge response in LDP.

Descriptors from each of the training images are used to form a bag of features, which was used to produce the training model. Next, a k-means clustering technique was applied on the patches from the training set for the generation of a codebook of the feature. The vocabulary size for the experiment was 1024. Descriptors from each training image are used with the code book to form a bag of features, to produce the training model.

\subsection{Fusion of the multi angle image}

The fusion of the multi angled images was performed in the both image level and feature label. An example of the image label fusion is given in figure 10 .

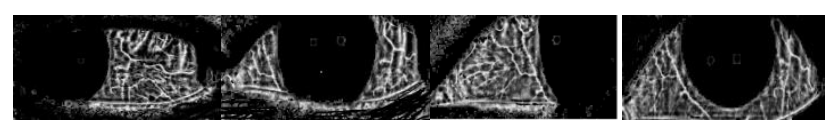

Figure 10.Image label fusion of different angles.

\subsection{Classification}

Support Vector Machines (SVMs) [23] are used over here for classification. A SVM is a popular supervised machine learning technique which performs an implicit mapping into a higher dimensional feature space for the classifiable data. The Library for Support Vector Machines (LIBSVM) was used here for SVM implementation. Though various new kernels are being proposed, the most frequently used kernel functions are linear, polynomial, and Radial Basis Function (RBF). This study uses the RBF kernel.

In the experiment we carried out grid-search on the hyper-parameters with 5-fold cross validation for selecting the parameters of the training sequence. The parameter settings that produce the best cross-validation accuracy were selected.

\section{Experimental Results}

The experimental setup and the results of our proposed work are explained in this section. They are aimed to evaluate the multi angle approach for anti-spoofing

\subsection{Data Set}

In order to evaluate the performance of the proposed method, an in house dataset was utilized in this experiment. This database consists of 5248 RGB images taken in one distinct session from 82 identities where each channel of RGB color space is represented in grey-scale. The individual comprise of both male and female and different color, few of them were wearing contact lens and images were taken in the different time in the day. The database contains blurred images and images with blinking eyes, closed eye and blurred eye images. High resolution images are provided in the database (300 dpi resolution and $7500 \mathrm{x}$ 5000 dimension). All the images are in JPEG format. Here for each individual image in four multi angle is considered. For each angle eight images are taken. For each individual both left and right eye is utilized. Different lighting conditions are considered during the image accusing. A NICON D 800 camera and 28300 lenses were used for image capturing. We have used different quality images and some of the sample images are shown below in Figure below.

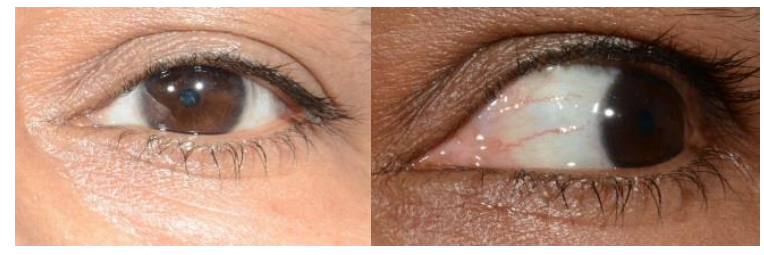

(a)

(b) 


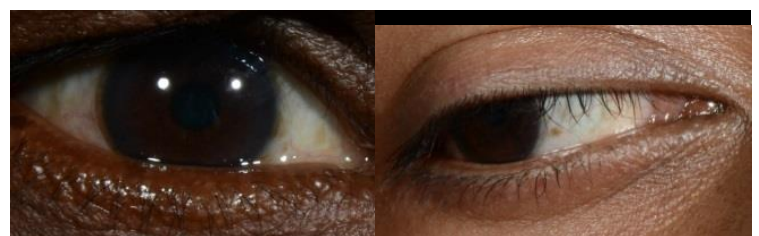

(c)

(d)

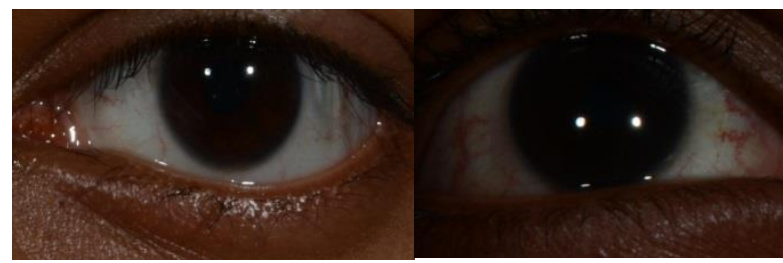

(e)

(f)

Figure 11: Different quality of eye images used.in the experiment

Some of them are not occluded having good quality of sclera regions visible, some of them are of medium quality and the third type was of poor quality with respect to sclera region visibility. In the experiments some closed eye image also used, examples of such images are provided below in Figure 12. There were 9 closed images and 3 blurred, these were added intentionally to add variation to the dataset and to experiment the tolerance of the recognition system w.r.t to sclera visibility and occlusion. The session images were taken in a dark room so that the noise factors such as reflection, luminosity, and contrast were minimized. The database contains blurred images and images with blinking eyes as shown in figure 12. In the experiments all the images of sessions 1 and 2 are considered. Here experiments were performed. For the experiment, sessions are considered separately; 4images set from each class are randomly chosen and utilized for training and the remaining 4 set images for testing performance.

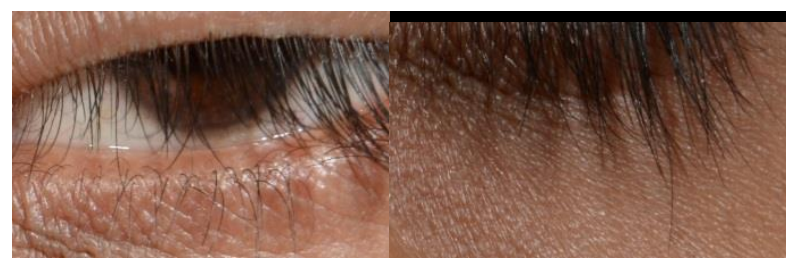

(a)

(b)

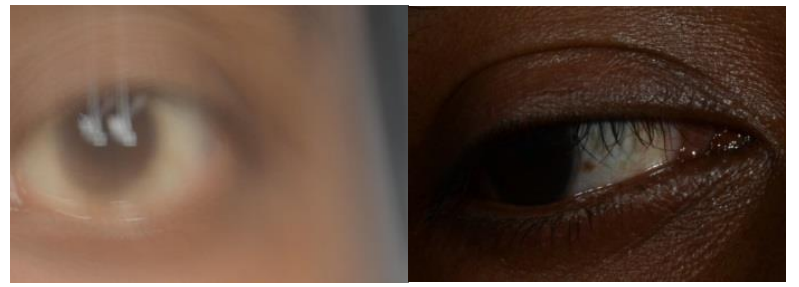

(c)

(d)

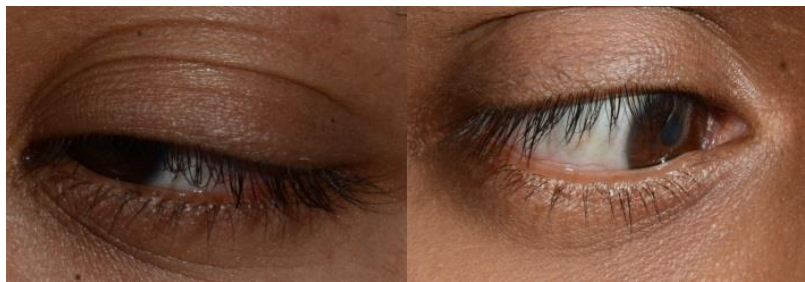

(d)

(e)

Figure 12: Example of closed and blurred eyes.

For experiment $164 * 4$ (we have 82 individual an both left and right eye of each individual is considered and pattern of each and every left and right eye is different for each individual) scores for FRR and 164*163 score for FAR statistics are achieved. All the simulation experiments performed here were developed in Matlab.

\subsection{Result Sclera Vessel Enhancement}

Experiment result of the different enhancement technique used for sclera vessel enhancement are discussed in this sub-section

\subsubsection{Image channel Selection}

In the experiments, it has been found that in the blue channel of the images, the sclera vessel patterns are most prominent as indicated in Figure 7.

\subsubsection{Preprocessing by Adaptive histogram equalization}

After that, adaptive histogram equalization was performed with a tiled window size of 42 X 42 at a clip limit of 0.01 , with full range and distribution exponential to get the best result.

\subsubsection{Preprocessing by Adaptive wavelet filter}

Further, Haar wavelet was used to enhance the vessel patterns. Low pass reconstruction with a cut off range of -0.65 and window size of $3 \times 3$.

\subsubsection{Preprocessing by Adaptive histogram equalization}

Next, the same adaptive histogram equalization with a tiled window size of $42 \times 42$ at a clip limit of 0.01 , with full range and distribution exponential is imposed on the filtered image.

\subsection{Time complexity}

The average time complexity result of segmentation, vessel enhancement, feature extraction and classification are given below in Table III. It can be inferred from the below table that the time complexity of the proposed technique 
was satisfactory. All the stimulated data reported here were developed in Matlab under Intel I5 processor in windows environment

TABLE I. TIME COMPLEXITY TABLE

\begin{tabular}{|c|c|}
\hline Different Steps & Time in Seconds \\
\hline Segmentation & 0.4 \\
\hline Vessel enhancement & 0.1 \\
\hline Feature extraction & 0.41 \\
\hline Classification & 0.05 \\
\hline
\end{tabular}

\subsection{Overall Experimental Results}

First we check the ability of the multi-angle scheme to verify the user identity. Table II show the Equal Error Rate (EER) of the device using different angles. It can be seen that the best angle is looking up although all of them get competitive results with the classical strategy of looking straight.

TABLE II. EQUAL ERROR RATE OF THE OVERALL RESULT USING THE DENSE LDP FEATURE IN DIFFERENT ANGLE

\begin{tabular}{|c|c|}
\hline Different Angle & EER (\%) \\
\hline Looking up & 0.9 \\
\hline Looking Left & 1.1 \\
\hline Looking right & 1.41 \\
\hline Looking stright & 1.05 \\
\hline
\end{tabular}

The results with forgeries are obtained by testing a high resolution picture with a wrong eye orientation (if the system is trained with straight orientation it is testes with left right and up orientation and so on). In this case, the ability of the system to reject such an input is given in Table III.

TABLE III. The results with forgeries obtained if the user is looking at different angle that the system have asked for

\begin{tabular}{|c|c|c|c|c|c|}
\hline & & \multicolumn{4}{|c|}{ Training } \\
\hline & looking & Up & Left & Right & Straight \\
\hline \multirow{3}{*}{ Testing } & Up & 0.9 & 20.12 & 17.88 & 19.56 \\
\cline { 2 - 6 } & Left & 19.75 & 1.1 & 21.99 & 14.66 \\
\hline
\end{tabular}

\begin{tabular}{|c|c|c|c|c|c|}
\hline \multirow{2}{*}{ Right } & 15.99 & 22.03 & 1.41 & 19.18 \\
\cline { 2 - 6 } & Straight & 19.55 & 15.01 & 19.08 & 1.05 \\
\hline
\end{tabular}

It can be reflected from the above table of each and every forgery angel the EER is high. Hence it can be concluded that this eye movement can work as good form for liveliness of data. The results of the different fusion levels are reflected in Table IV.

TABLE IV. EQUAL ERROR RATE OF THE DIFFERENT LEBEL OF FUSSION

\begin{tabular}{|c|c|}
\hline Different level of fusion & EER (\%) \\
\hline Image Label & 0.52 \\
\hline Feature label & 0.75 \\
\hline
\end{tabular}

It can be inferred from the above table that image label fusion produces the best results and the combination of different angle have increased the efficiency of the system.

Next to experiment the effect of sequence of the orientation that individual is looking i.e. if and individual is asked to look in a sequence of straight, up, left and right but the individual look in some other sequence, then what is the performance of the system. Table V and VI demonstrates the results of such example.

TABLE V. The results with forgeries obtained if the user is looking at different sequence of angles that the system have asked for by image level fusion

\begin{tabular}{|c|c|c|}
\hline & $\begin{array}{c}\text { Testing Sequence of } \\
\text { orientation }\end{array}$ & EER in \\
\hline & Straight, up, left and right & 0.52 \\
\hline & Straight, up, right and left & 19.90 \\
\hline & Straight, left, up and right & 17.01 \\
\hline & Straight, left, right and up & 23.05 \\
\hline & Straight, right, left and up & 24.01 \\
\hline & Straight, right, up and left & 22.09 \\
\hline & Right, left, up and straight & 24.35 \\
\hline inin & Right, left, straight and up & 22.01 \\
\hline $\begin{array}{c}\text { sequence } \\
\text { of } \\
\text { looking }\end{array}$ & Right, up, left and straight & 23.44 \\
\hline
\end{tabular}




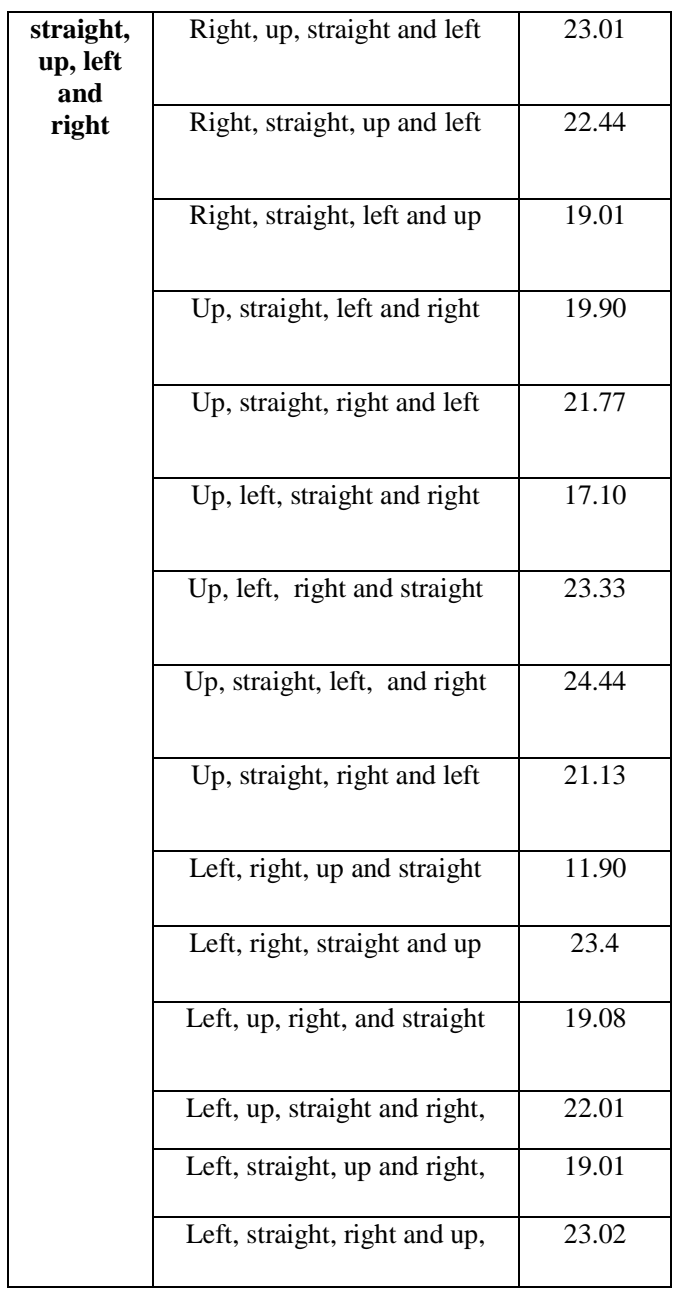

TABLE V. The results with forgeries obtained if the user is looking at different sequence of angles that the system have asked for by feature level fusion

\begin{tabular}{|c|c|c|}
\hline & Testing Sequence of orientation & EER in \% \\
\cline { 2 - 3 } & Straight, up, left and right & 0.75 \\
\cline { 2 - 3 } & Straight, up, right and left & 19.99 \\
\cline { 2 - 3 } & Straight, left, up and right & 19.09 \\
\cline { 2 - 3 } & Straight, left, right and up & 23.95 \\
\cline { 2 - 3 } & Straight, right, left and up & 24.81 \\
\hline & Straight, right, up and left & 22.39 \\
& Right, left, up and straight & 24.05 \\
\hline
\end{tabular}

\begin{tabular}{|c|c|c|}
\hline \multirow{17}{*}{$\begin{array}{c}\text { Training } \\
\text { by } \\
\text { sequence } \\
\text { of } \\
\text { looking } \\
\text { straight, } \\
\text { up, left } \\
\text { and } \\
\text { right }\end{array}$} & Right, left, straight and up & 22.81 \\
\hline & Right, up, left and straight & 23.94 \\
\hline & Right, up, straight and left & 23.81 \\
\hline & Right, straight, up and left & 22.49 \\
\hline & Right, straight, left and up & 19.01 \\
\hline & Up, straight, left and right & 19.99 \\
\hline & Up, straight, right and left & 21.8 \\
\hline & Up, left, straight and right & 17.90 \\
\hline & Up, left, right and straight & 23.77 \\
\hline & Up, straight, left, and right & 24.66 \\
\hline & Up, straight, right and left & 22.03 \\
\hline & Left, right, up and straight & 12.0 \\
\hline & Left, right, straight and up & 23.87 \\
\hline & Left, up, right, and straight & 19.99 \\
\hline & Left, up, straight and right, & 22.65 \\
\hline & Left, straight, up and right, & 19.88 \\
\hline & Left, straight, right and up, & 23.74 \\
\hline
\end{tabular}

It can be reflected from the above table of each and every forgery angel sequence the EER is high. Hence it can be concluded that this sequence of eye movement can work as good form for liveliness of data.

The experiment is extended by training with images sequence from the dataset and testing it with set of images captured by images of eye of the same set of individual from a mobile or portable screen (to stimulate a practical life scenario when eye image can be scanned for spoofing in front of a sensor by high resolution image).

The EER obtained in the result of the above mention experiment was 10.05. This signifies the robustness of the system to spoofing by portable devices. 
TABLE V1. EQUAL ERROR RATE OF THE DIFFERENT LEBEL OF FUSSION BY TESTING THE MOBILE IMAGES

\begin{tabular}{|c|c|}
\hline Different level of fusion & EER (\%) \\
\hline Image Label & 10.05 \\
\hline Feature label & 10.79 \\
\hline
\end{tabular}

\section{Conclusions}

An initiative is taken in this piece of work for liveliness in eye biometrics for contactless sclera biometric. The liveliness is implemented by asking the user to look at different random angle each time approach to the biometric scheme. Two advantages are obtained: $1^{\text {st }}$. we require a body response for aliveness detection, and $2^{\text {nd }}$. Looking to left, right or up the portion of sclera shown is different. So, we can require a different sclera angle in case of not being sure of user identity. Validation with respect to the single genuine angle and single forgery angle and sequence of forgery angle are also analysis in the work. The result with combination of different angle in different level of fusion was also analysis in the experiment. Satisfactory result was obtained in the experiment. Future scope will concentrates on designing liveliness based multi-modal eye biometrics combining sclera and iris traits.

\section{References}

[1] Z. Zhou, Y. Du, N. L. Thomas, and E. J. Delp. A new biometric sclera recognition. IEEE transaction on System, Man AndCybernatics -PART A: System And Human, 42(3): 571-583, 2012.

[2] J. G. Daugman. High confidence visual recognition of persons by a test of statistical independence. IEEE Transactions on Pattern Analysis and Machine Intelligence, 15(11):1148-1161, 1993.

[3] R. Derakhshani, A. Ross, and S. Crihalmeanu. A new biometric modality based on conjunctival vasculature. Proceedings of Artificial Neural Networks in Engineering: $1-8,2006$.

[4] Z. Zhou, Y. Du, N. L. Thomas, and E. J. Delp. Multi angled sclera recognition. IEEE Workshop on Computational Intelligence in Biometrics and Identity Management: 103 108, 2011.

[5] S. Crihalmeanu and A. Ross, Multispectral sclera patterns for ocular biometric recognition, Pattern Recognition Letters, 33: 1860-1869, 2012.

[6] A. Das, U. Pal, M. F. A. Ballester and, M. Blumenstein, A New Method for Sclera Vessel Recognition using OLBP, In Chinese Conference on Biometric Recognition ,LNCS 8232, 370-377,2013.

[7] M. A. Ferrer, A. Morales, A. Das, M. Blumenstein and Umapada Pal, "Model based Sclera vessels segmentation with SIFT Recognition and its combination with Iris", appeared in Spanish biometric consortum, VII Jornadas de
Reconocimiento Biometrico de Personas, Zamora, Spain, 68-76, 2013.

[8] A. Das, U. Pal, M. F. Ballester and M. Blumenstein, Sclera Recognition Using D-SIFT, In13th International Conference on Intelligent Systems Design and Applications74-79, 2013.

[9] A. Das, U. Pal, M. Blumenstein and M. F.Ballester, Sclera Recognition - A Survey, In Recent Advancement in Computer Vision and Pattern Recognition , 917 -921, 2013.

[10] B.N. Li, C.K. Chui, S. Chang, S.H. Ong, Integrating spatial fuzzy clustering with level set methods for automated medical image segmentation, Computers in Biology and Medicine 41(1) 1-10, 2011.

[11] Md. H. Kabir, T. Jabid, O. Chae, A Local Directional Pattern Variance (LDPv) based Face Descriptor for Human Facial Expression Recognition, In Proceedings of the IEEE Advanced Video and Signal Based Surveillance (AVSS), pp. 526-532, August 2010

[12] S. Lazebnik, C. Schmid, and J. Ponce, "Beyond bags of features: Spatial pyramid matching for recognizing natural scene categories," In Proc. Computer Vision and Pattern Recognition (CVPR), ( 2), 2169-2178, 2006.

[13] Z. Zhou, Y. Du, N. L. Thomas, and E. J. Delp, Multimodal eye recognition. Proceedings of the International Society for Optical Engineering, 7708(770806):1-10, 2010.

[14] Z. Zhou, Y. Du, N. L. Thomas, and E. J. Delp, Quality Fusion Based Multimodal Eye Recognition, IEEE International Conference on Systems, Man, and Cybernetics : 1297-1302, 2012.

[15] M. H. Khosravi and R.Safabakhsh, Human eye sclera detection and tracking using a modified time-adaptive self-organizing map, Pattern Recognition, 41: 2571 - 2593, 2008.

[16] S. P. Tankasala, P. Doynov, R. R. Derakhshani, A. Ross and S. Crihalmeanu, Biometric Recognition of Conjunctival Vasculature using GLCM Features, International Conference on Image Information Processing : 1-6, 2011.

[17] K. Oh and K.Toh, Extracting Sclera Features for Cancelable Identity Verification, 5th IAPR International Conference on Biometric: 245-250, 2012.

[18] V.Gottemukkula, S. K. Saripalle, S. P. Tankasala, R. Derakhshani, R. Pasula and A. Ross, Fusing Iris and Conjunctival Vasculature: Ocular Biometrics in the Visible Spectrum, IEEE Conference on Technologies for Homeland Security: 150-155, 2012.

[19] B.Toth, Biometric Liveness Detection, Information Security Bulletin, 10, 291-297, 2005.

[20] A. Das, U. Pal, M. F. Ballester and M. Blumenstein, Fuzzy Logic Based Sclera Recognition, In WCCI: 561-568, 2014.

[21] S. M. Pizer, E. P. Amburn and J. D. Austin, Adaptive Histogram Equalization and Its Variations, Computer Vision, Graphics, and Image Processing 39: 355-368, 1987.

[22] I. Daubechies, Ten lectures on wavelets, CBMS-NSF conference series in applied mathematics, SIAM Ed: 117119, 1992.

[23] H. William, A. Saul, T. William, B. P. Flannery, Support Vector Machines. Numerical Recipes: The Art of Scientific Computing (3rd ed.). New York: Cambridge University Press. ISBN 978-0-521-88068-8, 2007. 